\title{
The effect of Rosa canina L. and a polyherbal formulation syrups in patients with attention deficit/hyperactivity disorder: A study protocol for a multicenter randomized controlled trial
}

Haide Golsorkhi

Iran University of Medical Sciences

Mostafa Qorbani

Alborz University of Medical Sciences

Mohammad Kamalinejad

Shaheed Beheshti University of Medical Sciences

Saeideh Sabbaghzadegan

Iran University of Medical Sciences

Mohsen Bahrami

Private Clinic, Tehran, Iran

Mohammad Vafaee Shahi

Iran University of Medical Sciences

Hadi Montazerlotfelahi

Alborz University of Medical Sciences

Elham Abniki

Private clinic, Tehran, Iran

Majid Dadmehr ( $\triangle$ majiddadmehr@yahoo.com )

Iran University of Medical Sciences https://orcid.org/0000-0002-7032-8648

\section{Research Article}

Keywords: Attention deficit/hyperactivity disorder, ADHD, Complementary and Alternative Medicines, Herbal medicine

Posted Date: May 3rd, 2021

DOl: https://doi.org/10.21203/rs.3.rs-345669/v1

License: (a) This work is licensed under a Creative Commons Attribution 4.0 International License. Read Full License 


\section{Abstract}

Background: Attention deficit/hyperactivity disorder (ADHD) is the most common behavioral disorder in childhood and adolescence. A number of these patients do not respond to the current pharmacological treatments and there may also be drug side effects. This study aims to determine the efficacy and safety of two herbal medicine products, including Rosa canina L. (RC) and a polyherbal formulation (PHF) syrups on the clinical manifestations of ADHD in children and adolescents.

Methods: Ninety ADHD patients based on DSM- 5 diagnostic criteria will be randomly assigned equally to three groups: (1) RC syrup + methylphenidate (MP); (2) PHF syrup + MP; (3) placebo + MP according to the inclusion criteria (30 subjects in each group). The syrups dosage is $5 \mathrm{cc}$ every 8 hours and MP has a stabilized dose for 8 weeks during the study. Moreover, Conner's questionnaires will be completed by the teacher and parents before the intervention and then every 4 weeks. Also, the child symptom inventoryfourth edition (CSI-4) and temperament questionnaires will be completed before the intervention and every four weeks until two months.

Discussion: This trial is the first experiment to determine the effects of RC and PHF syrups on the clinical manifestations of ADHD in children and adolescents. Our findings provide new insight into the effect of these herbal products on the clinical manifestations of ADHD.

Trial registration: The trial was registered at https://en.irct.ir/ (Registration number: IRCT20190923044855N1). Registration date: 2020-01-14.

\section{Background}

Attention deficit/hyperactivity disorder (ADHD) belongs to subset of neuropsychiatric disorder in children and adolescents, which is described by persistent impairing symptoms of inattention, hyperactivity, and impulsivity [1]. More than half of these patients, exhibit these symptoms during adulthood [2-4]. This disorder is frequently combined with learning problems, inability the psychosocial functioning also family, and public health problems $[1-3,5,6]$. ADHD carries a high risk of comorbidities such as anxiety, affective disorders, substance abuse, and personality disorders, also poor education consequences, a great chance for inoccupation, divorcement or imprisonment are more diagnosed among them compared to the general population $[3,5]$. Moreover, mood disorder is reported in $15-75 \%$ of them and $25 \%$ have anxiety [7]. The prevalence rate of ADHD in children is approximately $8-12 \%$ all over the world [3]. Treatment strategies for ADHD mainly include drug therapy, psychological and behavioral therapy. Psychostimulant drugs, especially methylphenidate (MP) are highly efficacious and the primary choice of pharmaceutical medications for $\operatorname{ADHD}$ treatment $[3,6,7]$. Stimulant medication effectively improves ADHD symptoms in most of these patients; however, about $30 \%$ of them do not respond to its pharmacological treatments, and there may also be drug side effects such as loss of appetite, sleep disturbances and anxiety; therefore, many parents seek complementary and alternative medicines (CAMs) such as food recommendations, herbal medicine, homeopathy, and exercise for controlling 
overactivity and inattention of ADHD [7]. Herbal medicine is the most commonly administered CAMs method for ADHD due to its well-tolerance and also parent's acceptance [8,9]. There are several mechanisms of action for these medicinal plants associated with the pathogenesis of ADHD, including anxiolytic and antidepressant effects, improvement of cognitive function that help to increase serotonin levels and central stimulating [7]. Although limited information has been reported on the beneficial effects of these plants, clinical studies are needed to support their efficacy and safety.

Rosa canina L. (RC) belongs to Rosacea family, which its different parts such as roots, leaves, and fruits have been used to treat several diseases for centuries in traditional medicine [10]. Studies have shown that RC has neuroprotective effects when used in combination with other herbs [11, 12]. Moreover, it has demonstrated that RC extract has anxiolytic properties and can improve recognition memory and depressive-like behavior $[13,14]$. In an experimental animal model, it is shown that chronic administration of Malus domestica Borkh. fruit juice has valuable antidepressant activity [15]. Ocimum basilicum L. is an edible herb, which has anxiolytic, sedative, and antidepressant-like effects [16, 17]. Also, its neuroprotective properties, especially the improvement of memory and neurological deficit have been documented previously [18]. Pharmacological studies displayed that Vitis vinifera L. has beneficial effects on cognitive function and neuropsychological status; additionally, indicates anxiolytic-like activity $[19,20]$. Therefore, we hypothesized that a polyherbal formulation (PHF) syrup contains Malus domestica Borkh., Ocimum basilicum L. and Vitis vinifera L. extract may have beneficial effects on ameliorating memory and cognitive function, and anxiety in children with ADHD.

This randomized clinical trial aim to determine the efficacy and safety of RC and PHF syrups on the clinical manifestations of ADHD in children and adolescents.

\section{Methods}

\section{Study design}

This study is a double-blind randomized clinical trial (two arms factorial design) to determine the efficacy of two herbal products in children with ADHD. The study protocol was reviewed and approved by the Medical Ethics Committee of Iran University of Medical Sciences [session no: IRIUMS.REC. 1398.561], and registered in the Iranian Registry of Clinical Trials [registration code: IRCT20190923044855N1]. Figure 1 provides details of the study schedule.

\section{Study population}

The patients will be mainly recruited from the children and adolescents aged 5 to 14 years who refer to the outpatient clinics of neurology and psychiatry of the study centers (at the Rasoul Akram academic hospital, the Firooz Abadi academic hospital and community clinics, Tehran, Iran) for diagnosis and treatment of ADHD. Those who meet the inclusion criteria will enroll in the trial.

\section{Eligibility criteria}


Patients will enroll in this trial if they have these criteria:

1) Male and female children aged 5 to 14 years; 2) Parents and children willing and able to follow all study visits; 3 ) Diagnosis of ADHD, according to DSM-5 diagnostic criteria; 4) Treatment with a stabilized dose of oral stimulant medications (e.g., MP) through the study period; 5) Non-use of other alternative and complementary medications that may interact with the herbal product.

\section{Exclusion criteria}

Children with any of the following criteria will be excluded from the trial:

1) History of mental retardation; 2) History of bipolar disorder, psychosis, severe conduct disorder, autism;

3) History of neurological diseases, seizures or other serious medical conditions.

\section{Withdrawal criteria}

The study withdrawal criteria are listed as follows:

1) Parents' or children's unwillingness to continue treatment or move to another location; 2) Possible side effects of treatment; 3 ) Adding another illness or not taking or misusing the drug.

Sample size calculationAccording to the previous studies, the mean \pm SD of ADHD questionnaires and methods mean deviation in three groups were 6.5 and 6, respectively. The sample size was 18 patients per group with a $\mathrm{Cl}$ of $95 \%$, power of $80 \%$, and loss of $15 \%$. A total of 90 patients will be invited and divided into three equal groups by using the block randomization method.

\section{Randomization}

The subjects who meet the eligibility criteria will be randomly divided into the intervention and placebo groups using permuted block randomization method. Moreover, stratified randomization will be used to match the subjects based on the age distributions (5-10 and 10-14 years old). Participants will be randomly assigned to three treatment groups of (1) RC syrup + MP; (2) PHF syrup + MP; (3) placebo + MP. Then they will be followed up for 8 weeks. Both investigators and participants will be blinded to the study design.

\section{Intervention}

RC, PHF and placebo are in the form of syrup and will be provided by the Sanabel Darou Co., Tehran, Iran. RC contains Rosa canina L. extract ((Vardibel $\left.{ }^{\mathrm{TM}}\right)$; PHF contains Malus domestica Borkh., Ocimum basilicum L. and Vitis vinifera L. extract (Pardihan ${ }^{\mathrm{T}}{ }^{\mathrm{M}}$ ); and placebo is considered sucrose.

Ninety children and adolescents with ADHD will be invited to the study and randomly assigned to three groups. Subjects will be required to consume $5 \mathrm{cc} /$ day every eight hours according to the recommended dosage for children in the syrup brochure. 
The syrups will be prepared in $250 \mathrm{cc}$ glassware and will suffice for about a month. The syrups are identical in size, color, and shape. Any possible complications regarding the numbers of syrups and package will be recorded. Also, the study progress will be pursued by recruiting the subjects every four weeks. Details of the study protocol (SPIRIT flowchart) are provided in Figure 2.

\section{Adherence}

To evaluate the compliance of the patients, they will be called every 4 weeks. At the first visit, the participants will receive interventions and will be asked to bring all eaten ones every month. Returned supplements will be observed to evaluate the level of compliance and adherence to the intervention.

\section{Patient safety}

All participants will be monitored and any probable adverse events will be reported during the study period.

\section{Study outcomes}

\section{Primary outcomes}

The primary objective of this research is to determine the efficacy and safety of these herbal medicine products on clinical manifestation of ADHD in children and adolescents.

\section{Secondary outcomes}

The secondary outcome of this study includes the changes in attention and activity function levels, socioeducational function levels, sleep, appetite and comorbidity (e.g. anxiety, depression, and obsessivecompulsive disorders) at the end of the study in comparison with the baseline values.

\section{Procedure}

At the beginning of the study, goals, methods, and benefits of the trail will be clarified to the parents and participants and an informed consent form will be provided to them. Conners Comprehensive Behavior Rating Scales (Conners CBRS) will be used for obtaining information about several important domains of participant's behavior, including behavioral, communal, educational topics, and their symptoms. In this study, two of Conner's CBRS questionnaires will be completed (both parent and teacher rating scales):

1- Conners' Parent Rating Scale (CPRS); a tool for effectively collecting parental reports of child behavior problems

2- Conners' Teacher Rating Scale (CTRS); a tool for obtaining teacher reports of children's behavior in the classroom [21-24].

Also, Child Symptom Inventory-4 (CSI-4) questionnaire will be used to evaluate attention problems in children. It is a behavior rating scale that is used for diagnosis and also severity assessment of ADHD in 
children between 5 and 12 years old $[25,26]$. Moreover, another questionnaire is temperament determinant questionnaire [27].

These questionnaires will be completed before the intervention and then only Conner's questionnaires will be used every 4 weeks by teacher and parents during the study. The interviewer will evaluate children signs and symptoms every 4 weeks for two months. There is no specific intervention to change the participants' lifestyle (diet, sleep, activity and etc.).

\section{Lifestyle changes}

No intervention is made in the patient's living conditions to evaluate the drug's efficacy.

\section{Data analysis}

The symptoms of hyperactivity/inattention are the most important indicator to investigate. Other indicators rank secondary importance. Descriptive statistics for qualitative variables include frequency tables, appropriate graphs. In addition, Chi-square or Fisher's exact tests will be used to investigate the relationship between the two qualitative variables. Regression imputation method will be used where the value of that variable is missing. The mean and standard deviation (SD) will be reported for quantitative variables and percentages for qualitative variables. ANOVA and Chi-square tests will be used in the three groups to compare quantitative and qualitative variables. A Cl of $95 \%$ will be applied to all the tests. The significance level will be considered less than 0.05 . Finally, SPSS 21 statistical software will be used to analyze the data.

\section{Data accessibility}

The final trial dataset will be only available to the principal investigator, and others investigators will have limited access. Finally, the study results will be presented only in the publication.

\section{Discussion}

To the best of our knowledge, this is a novel multicenter study designed for the first time to evaluate the therapeutic effects of two herbal formulations on the treatment of children and adolescents with ADHD and also their comorbidity, appetite and sleep.

The long-term use and side effects of conventional medications are a concern for parents, so they are increasingly looking for treatment options of CAMs [7]. A number of patients with ADHD diagnosis are frequently used CAMs, including dietary modifications, nutritional supplementation, and herbal medicine alone or along with current pharmacological treatments in order to manage of hyperkinetic and concentration disorders [2]. Diet, exercise and nutritional supplements all have some potential benefits for a child with ADHD. Herbal remedies, which have been shown to have good effects on restlessness, anxiety, and depression can also be proper options; however, more research is needed [7]. Moreover, the 
role of chronic inflammation and oxidative stress has been noted in ADHD. Dietary polyphenols have antioxidant and immunomodulatory properties; so, they may be useful in the management of ADHD [28].

Different herbal preparations have been evaluated in clinical trials as a treatment for children and adolescents with ADHD. The findings propose that some of them may be as effective as MP [6]. RC has some beneficial effects on anxiety, recognition, memory and, depressive-like behaviors $[13,14]$. Numerous studies have shown that the ingredients in PHF syrup have considerable ameliorating effects on anxiety, depression and memory [15-20]. So, the authors hypothesized that RC and PHF syrups will be valuable for the management of ADHD.

\section{The Strengths And Limitations}

Our study has several strengths such as providing a randomized double-blinded design and protocol publication. Currently, no side effects and drug interactions are reported on selected herbs in the related review articles. The limitations of this study include lack of cooperation of some participants to complete the intervention (child or parents unintentional for continuing intervention).

\section{Abbreviations}

ADHD: Attention deficit/hyperactivity disorder; DSM-5: Diagnostic and statistical manual of mental disorders; CSI-4: Child symptom inventory-fourth edition; CAMs: Complementary and alternative medicines; SD: Standard deviation; Cl: Confidence interval; Conners CBRS: Conners Comprehensive Behavior Rating Scales; CTRS: Conners' Teacher Rating Scale; CPRS: Conners' Parent Rating Scale

\section{Declarations}

\section{Ethics approval and consent to participate}

The Ethics Committee of Iran University of Medical Sciences has approved study protocol [session no: IRIUMS.REC. 1398.561]. At the beginning of the study, a written informed consent form will be obtained from a parent or guardian for participants under 16 years old.

\section{Consent for publication}

Not applicable.

\section{Availability of data and material}

Not applicable.

\section{Competing interests}

The authors declare that they have no competing interests. 


\section{Funding}

This research has been supported by Iran University of Medical Sciences (grant no. 98-2-63-15165). The funder has no role in study design, collection, analysis, and interpretation of data, writing of the paper and the decision to submit the paper.

\section{Authors' contributions}

$M B$ and MK designed the idea of this work. HG, MD and MVS coordinated the study. MQ advised on statistical analysis. HG, HM, MVS and EA organized participant management and data collection. HG, MD and SS drafted the manuscript. The manuscript has been read and approved by all authors.

\section{Acknowledgements}

Not Applicable

\section{References}

1. Polanczyk G, et al. The worldwide prevalence of ADHD: a systematic review and metaregression analysis. American journal of psychiatry. 2007;164(6):942-8.

2. Sarris $\mathrm{J}$, et al. Complementary medicines (herbal and nutritional products) in the treatment of Attention Deficit Hyperactivity Disorder (ADHD): a systematic review of the evidence. Complement Ther Med. 2011;19(4):216-27.

3. Luo Y, et al. A review of heterogeneity in attention deficit/hyperactivity disorder (ADHD). Front Hum Neurosci. 2019;13:42.

4. Sharma A, Couture J. A review of the pathophysiology, etiology, and treatment of attention-deficit hyperactivity disorder (ADHD). Ann Pharmacother. 2014;48(2):209-25.

5. Lee SS, et al. Prospective association of childhood attention-deficit/hyperactivity disorder (ADHD) and substance use and abuse/dependence: a meta-analytic review. Clin Psychol Rev. 2011;31(3):328-41.

6. Wong YW, Kim D-g, Lee J-y, Traditional oriental herbal medicine for children and adolescents with ADHD: a systematic review. Evidence-Based Complementary and Alternative Medicine, 2012. 2012.

7. Pellow J, Solomon EM, Barnard CN. Complementary and alternative medical therapies for children with attention-deficit/hyperactivity disorder (ADHD). Altern Med Rev. 2011;16(4):323-37.

8. Shakibaei F, et al. Ginkgo biloba in the treatment of attention-deficit/hyperactivity disorder in children and adolescents. A randomized, placebo-controlled, trial. Complement Ther Clin Pract. 2015;21(2):61-7.

9. Gromball J, et al. Hyperactivity, concentration difficulties and impulsiveness improve during seven weeks' treatment with valerian root and lemon balm extracts in primary school children. Phytomedicine. 2014;21(8-9):1098-103. 
10. Selahvarzian A, et al. Medicinal Properties of Rosa canina L. Herbal Medicines Journal. 2018;3(2):77-84.

11. Daneshmand $P$, et al. Neuroprotective Effects of Herbal Extract (Rosa canina, Tanacetum vulgare and Urtica dioica) on Rat Model of Sporadic Alzheimer's Disease. Avicenna J Med Biotechnol. 2016;8(3):120-5.

12. Vafaee F, et al. Neuroprotective effects of the immunomodulatory drug Setarud on cerebral ischemia in male rats. Neural Regen Res. 2012;7(27):2085-91.

13. Farajpour R, et al. Chronic Administration of Rosa canina Hydro-Alcoholic Extract Attenuates Depressive-Like Behavior and Recognition Memory Impairment in Diabetic Mice: A Possible Role of Oxidative Stress. Med Princ Pract. 2017;26(3):245-50.

14. Nemati Z, et al. Effect of a hydroalcoholic extract of Rosa Canina flowers on anxiety in rats. Neurophysiology. 2015;47(2):133-7.

15. Lobo AR, Satish S. An investigation on anti-depressant activity of fresh fruit juice of Malus domestica in experimental animal models. 2019.

16. Rabbani M, Sajjadi SE, Vaezi A. Evaluation of anxiolytic and sedative effect of essential oil and hydroalcoholic extract of Ocimum basilicum L. and chemical composition of its essential oil. Research in pharmaceutical sciences. 2015;10(6):535.

17. Ali SS, et al. The antidepressant-like effect of Ocimum basilicum in an animal model of depression. Biotech Histochem. 2017;92(6):390-401.

18. Singh V, Krishan P, Shri R. Improvement of memory and neurological deficit with Ocimum basilicum L. extract after ischemia reperfusion induced cerebral injury in mice. Metabolic brain disease. 2018;33(4):1111-20.

19. Aslam M, Sultana N. Evaluation of anxiolytic-like activity of Vitis vinifera juice in mice. Avicenna $J$ Phytomed. 2016;6(3):344-50.

20. Calapai G, et al. A Randomized, Double-Blinded, Clinical Trial on Effects of a Vitis vinifera Extract on Cognitive Function in Healthy Older Adults. Front Pharmacol. 2017;8:776.

21. Smyth AC, Meier ST. Evaluating the psychometric properties of the conners adult ADHD rating scales. J Atten Disord. 2019;23(10):1111-8.

22. Izzo VA, Donati MA, Primi C. Assessing ADHD through the multi-informant approach: The contribution of the Conners'3 scales. J Atten Disord. 2019;23(6):641-50.

23. Abdekhodaie Z, Tabatabaei SM, Gholizadeh M. The investigation of ADHD prevalence in kindergarten children in northeast Iran and a determination of the criterion validity of Conners' questionnaire via clinical interview. Res Dev Disabil. 2012;33(2):357-61.

24. Shaked D, et al., Reliability and validity of the Conners' continuous performance test. Applied Neuropsychology: Adult, 2019: p. 1-10.

25. Shooshtary $\mathrm{MH}$, et al. The prevalence of attention deficit hyperactivity disorder in Iran: A systematic review. Iranian journal of psychiatry. 2010;5(3):88. 
26. Mohammad Esmaeel E. Adaptation and standardization of child symptom inventory-4 (CSI-4). Journal of Exceptional Children. 2007;7(1):79-96.

27. Salmannezhad $\mathrm{H}$, et al., Design and Validation of Mizaj Identification Questionnaire in Persian Medicine. Iranian Red Crescent Medical Journal, 2018. 20(11).

28. Verlaet AA, et al. Rationale for dietary antioxidant treatment of ADHD. Nutrients. 2018;10(4):405.

\section{Figures}

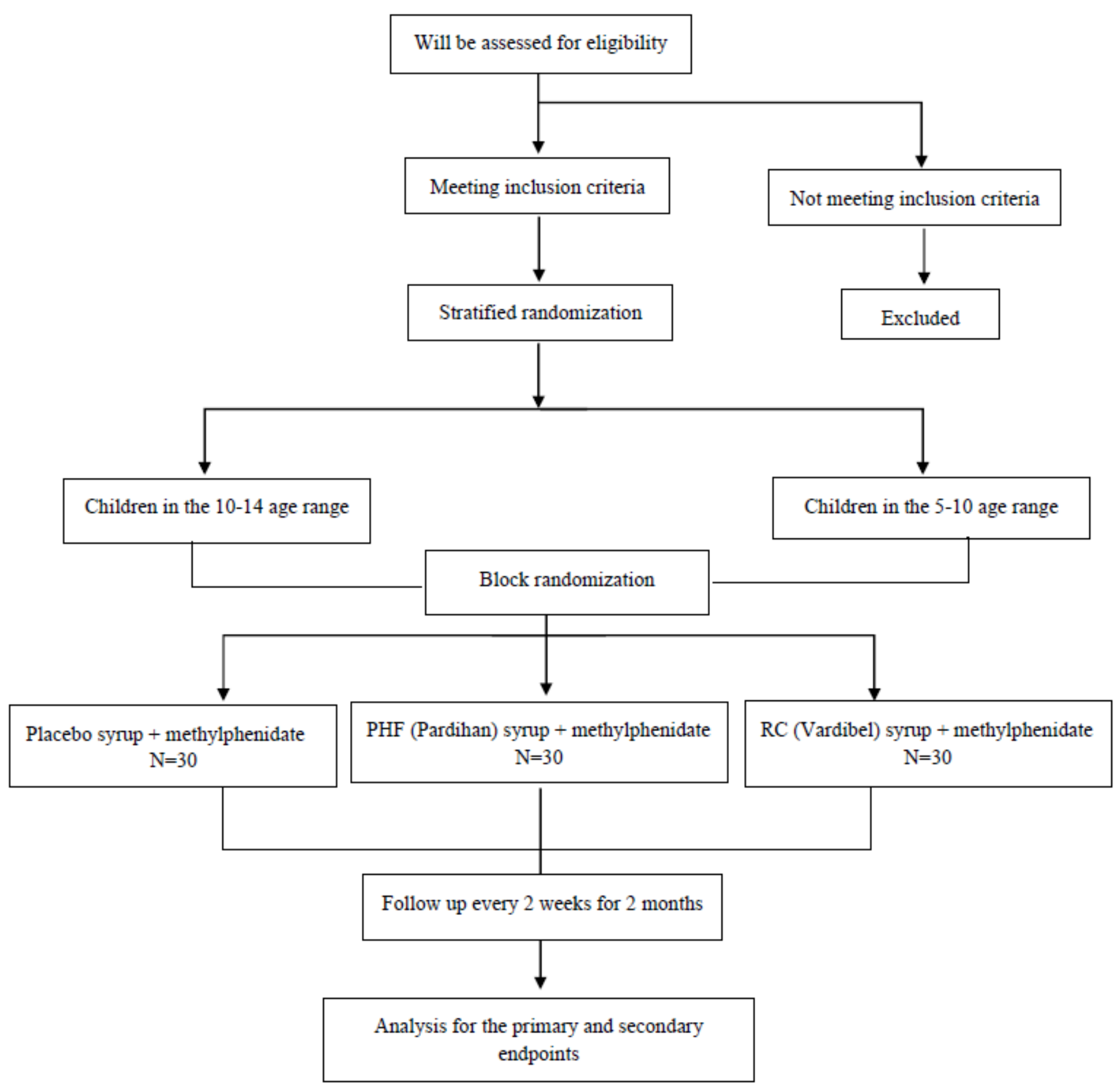


CONSORT Flow Diagram of the study

\begin{tabular}{|l|c|c|c|}
\hline Assessments & Baseline $\left(\mathbf{1}^{\text {st }}\right.$ Week) & $\mathbf{4}^{\text {th }}$ Week & End of the study (8 ${ }^{\text {th }}$ Week) \\
\hline Enrollment (inclusion/exclusion criteria) & $*$ & & \\
\hline Written informed consent (signed and dated) & $*$ & & \\
\hline Randomization & $*$ & & \\
\hline $\begin{array}{l}\text { General demography and relevant medical } \\
\text { history }\end{array}$ & $*$ & $*$ & $*$ \\
\hline Physical examination & $*$ & $*$ & $*$ \\
\hline Current medication use & $*$ & $*$ & $*$ \\
\hline Conners' Parent Rating Scale & $*$ & $*$ & $*$ \\
\hline Conners' Teacher Rating Scale & $*$ & & $*$ \\
\hline Child Symptom Inventory-4 (CSI-4) questionnaire & $*$ & $*$ & \\
\hline Temperament determinant questionnaire & $*$ & $*$ \\
\hline Compliance of intervention & $*$ & & \\
\hline
\end{tabular}

Figure 2

Standard Protocol Items Recommendations for Interventional Trials (SPIRIT) flowchart of the study.

\section{Supplementary Files}

This is a list of supplementary files associated with this preprint. Click to download.

- SPIRITChecklist17.3.2021.doc 